\title{
NOVEL APPROACHES IN THE CLASSIFICATION AND RISK ASSESSMENT OF PATIENTS WITH MYELODYSPLASTIC SYNDROMES-CLINICAL IMPLICATION
}

\author{
Ilina Micheva ${ }^{1}$, Rosen Rachev ${ }^{1}$, Hinco Varbanov ${ }^{1}$, Vladimir Gerov², Liana Gercheva ${ }^{1}$ \\ ${ }^{1}$ Hematology Division, Department of Internal Medicine, Medical University of Varna, \\ ${ }^{2}$ Clinic of Hematology, University Hospital "Sveta Marina", Varna
}

\begin{abstract}
INTRODUCTION: New prognostic systems have been proposed, aimed at improving the ability to predict survival and progression in myelodysplastic syndome (MDS) patients, as well as to select the accurate therapy.

PURPOSE: The aim of this study was to determine the prognostic score for patients with MDS, diagnosed in the Hematology Clinic, University hospital, Varna, comparing different prognostic scoring systems.

MATERIALS AND METHODS: 92 patients with MDS, diagnosed between 2004 and 2012 were included in the study. The median age was 72 (20-91 years), 87\% >60 years of age, 52 men and 41 women. The prognostic score was determined according to IPSS, WPSS, MDACSS. The parameters assessed were WHO type, karyotype, cytopenias, percentage of marrow blasts, age, performing status and transfusion dependence.

RESULTS: Cytogenetic studies were performed in $100 \%$ of patients. Abnormalities were found in $34.8 \%$, the most common-del-5q (14.6\%), -Y (4.5\%), complex karyotype (8\%), +8 (2.2\%). Patients were distributed according to the cytogenetic risk -low-87.2\%, intermediate-7.7\%, high-5.1\%. According to IPSS $41 \%$ of the patients were with low risk, 34\%-Int-1, 16\%-Int-2, high-9\%. WPSS distributed the patients in 5 groups: very low- $11 \%$, low risk- $43 \%$, int- $21 \%$, high-20\%, very high-5\%. According to MDACSS $14 \%$ of the patients were with low, 54\%-Int-1, $18 \%$-Int 2, $13 \%$ with high risk.

CONCLUSION: Significant correlation was found between the risk groups and AML transformation and survival. However, the comparison between the different scoring systems demonstrated the advantage of prognostic systems, based on a broader range of clinical parameters in better distribution of intermediate risk patients and the precise determination of high and very high risk.
\end{abstract}

Keywords: myelodysplastic syndrome, classification, prognostic systems

Address for corespondence

Ilina Micheva, MD, PhD

Clinic of Hematology

St. Marina University Hospital of Varna,

1 Hristo Smirnenski Str., 9010 Varna, Bulgaria

Medical University of Varna

email: ilinamicheva@gmail.com

Received: October 10, 2013

Accepted: December 18, 2013 


\section{INTRODUCTION}

Myelodysplastic syndromes (MDS) are clonal disorders of hematopoiesis characterised by ineffective hematopoiesis, peripheral blood cytopenias, and risk of progression to acute myeloid leukemia (AML). The clinical phenotype of patients with MDS is diverse with respect to the number and severity of cytopenias, cellularity and blast count in the bone marrow, rate of progression to AML, overall survival, and response to treatment.

Staging systems are a key to accurate MDS diagnosis and selection of therapy. MDS staging and classification schemes have evolved significantly over the past few decades to address our understanding of the biology of this disease. A variety of clinical and morphological issues led to the development of a new MDS staging system by the World Health Organization (WHO) to further refine the classification criteria and improve the assignment of prognosis for intermediate-risk cases (3).

In the summer of 2008 a revised version of WHO syllabus was published (10). For the first time the $2008 \mathrm{WHO}$ classification proposed a diagnosis of "presumptive MDS" in the case of persistent clinical cytopenias without dysplasia if certain cytogenetic abnormalities are present. The term Idiopathic cytopenia of undetermined significance (ICUS) is applied if the patients have persistent cytopenias without alternative explanation, no dysplasia and specific cytogenetic abnormalities. Refractory cytopenia with unilineage dysplasia (RCUD) includes cases where dysplasia is demonstrated in $10 \%$ of one cell line. The majority of cases with fibrosis are RAEB and are associated with a worse outcome. For CMML the diagnostic requirements remain the same. A subset of patients with CMML and eosinophilia, associated with genetic abnormalities including platelet derived growth factor receptor (PDGFR), are better classified as myeloid neoplasms with eosinophilia. Provisional entity refractory anemia with ringed sideroblasts associated with marked thrombocytosis (RARS-T) is proposed where up to $60 \%$ of patients harbour the JAK2 V617F mutation. The term myeloproliferative disorders was replaced by myeloproliferative neoplasms.
Recently several novel prognostic systems have been proposed, aimed at improving the ability to predict survival and progression in MDS patients.

The most widely used MDS prognostic system in clinical practice continues to be the International Prognostic Scoring System (IPSS) (4), but clinicians have recognised for many years that IPSS has a number of clinical limitations. These limitations include unsatisfactory applicability of IPSS to a variety of clinical situations such as secondary, therapyrelated MDS or previously treated patients; lack of sensitivity to the degree of patients' peripheral blood cytopenias, especially severe thrombocytopenia; excessive emphasis on elevated marrow blast proportion compared to high risk cytogenetics; a restricted number of included karyotypes; and failure to account for either comorbid conditions, or newer biomarkers of demonstrated prognostic value such as the high grade marrow fibrosis, or elevated serum ferritin level. Collectively these limitations made the original IPSS a less accurate and helpful tool and prompted other investigators to derive and propose newer risk stratification tools. Malcovati et al (8) developed a dynamic prognostic model based on the variables introduced by the WHO Classification-Based Prognostic Scoring System (WPSS). The WPSS score integrates the three most important prognostic factors in MDS patients classified according to WHO criteria: karyotype, WHO subgroup, and requirement for red blood cell (RBC) transfusion. This model provides dynamic prognostic information throughout the clinical course estimating the relationship between variables repeatedly measured during the follow-up. The WPSS stratifies MDS patients into five different risk categories: very low, low, intermediate, high, or very high. Significant differences are seen among the five groups in overall survival and risk of AML. In 2008, Kantarjian and colleagues from MD Andersen Cancer Center (7) proposed a 4-strata generalised risk model for patients with MDS- MD Andersen Cancer Center risk model (MDACSS). MDACSS is both sensitive to the degree of cytopenias and emphasises the importance of high-risk karyotype, which as several analyses have suggested is a more powerful marker of a negative outcome than increased marrow blast proportion. A multivariate analysis of 
prognostic factors in the study group identified the following adverse, independent factors as continuous and categorical values $(\mathrm{P}<.001)$ : poor performance, older age, thrombocytopenia, anemia, increased bone marrow blasts, leukocytosis, chromosome 7 or complex ( $\geq 3$ ) abnormalities, and prior transfusions. Cutoffs for anemia, thrombocytopenia and blasts, and cytogenetic subsets were different according to the IPSS. The new MDS prognostic model divided patients into 4 prognostic groups with significantly different outcomes.

The cytogenetic profile in patients with MDS remains the most powerful independent prognostic marker. The cytogenetic risk is determined according to the IPSS criteria. Recently a much broader range of karyotypes than the initial IPSS (based on the German-Austrian consortium) was analysed and patients were distributed in 4 cytogenetic risk groups (6). Cytogenetic analyses in 2072 patients revealed clonal abnormalities in $52.3 \%$. Karyotypes involving deletions of $5 \mathrm{q}$ were the most frequent, occurring in $30 \%$ of the patients with clonal cytogenetic abnormalities. Other frequent anomalies were $-7 / \operatorname{del}(7 q)(21 \%),+8$ (16\%), $-18 / 18 q-(7 \%)$, 20q- (7\%), -5 (6\%), -Y (5\%), -17/17p- (including isochromosome (17q)) (5\%), +Mar (5\%), +21 (4\%), inv/t(3q) (4\%), -13/13q- (4\%), +1/+1q (3\%), -21 (3\%), $+11(3 \%), 12 p-(2 \%), t(5 q)(2 \%), 11 q-(2 \%)$, and $\mathrm{t}(7 \mathrm{q})$ (2\%). For all World Health Organization (WHO) and French-American-British (FAB) classification system subtypes, the karyotype provided additional prognostic information.

All the advantages of the new scoring systems were incorporated in the revised IPSS (R-IPSS) first discussed in May 2011 by Greenberg at the 11th MDS Foundation International Symposium in Edinburgh, Scotland and later published in 2012 (5). The basic parameters of R-IPSS are the bone marrow cytogenetics, marrow blast percentage, and cytopenias. The novel components of the system included: 5 rather than 3 cytogenetic prognostic subgroups with specific and new classifications of a number of less common cytogenetic subsets (9), splitting the low marrow blast percentage value, and depth of cytopenias. This model defines 5 rather than the 4 major prognostic categories that are present in the IPSS. This system comprehensively integrated the numerous known clinical features into a method analysing MDS patient prognosis more precisely than the initial IPSS.

A future direction in the development of new risk stratification systems is the incorporation of novel prognostic markers such as the somatic mutations found in MDS (1). The somatic gene mutations landscape continues to expand and none of the existing risk models incorporate additional analysis. Bejar et al (2) examined whether the mutation status for each gene was associated with clinical variables, including specific cytopenias, the proportion of blasts, and overall survival. They found that mutations in EZH2, ASHL2, TP53, RUNX1 all conferred an IPSS independent adverse prognosis in terms of overall survival. DNMT3A mutations are also associated with strikingly adverse negative IPSS and karyotype independent prognostic effect on both MDS and AML (11).

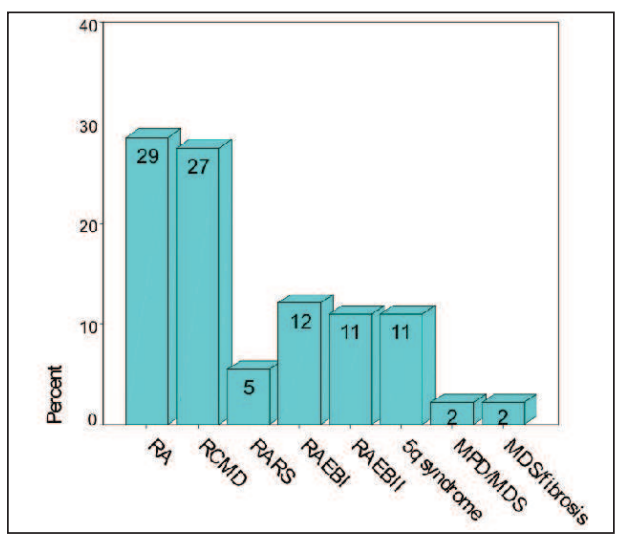

Fig. 1. Distribution of MDS patients according to WHO classification

The aim of this study was to classify and determine the prognostic score for patients with MDS, diagnosed in the Hematology Clinic, University hospital, Varna, comparing different prognostic scoring systems.

\section{PATIENTS AND METHODS}

92 patients with MDS, diagnosed between 2004 and 2012 were included in the study. The 
Ilina Micheva, Rosen Rachev, Hinco Varbanov et al.

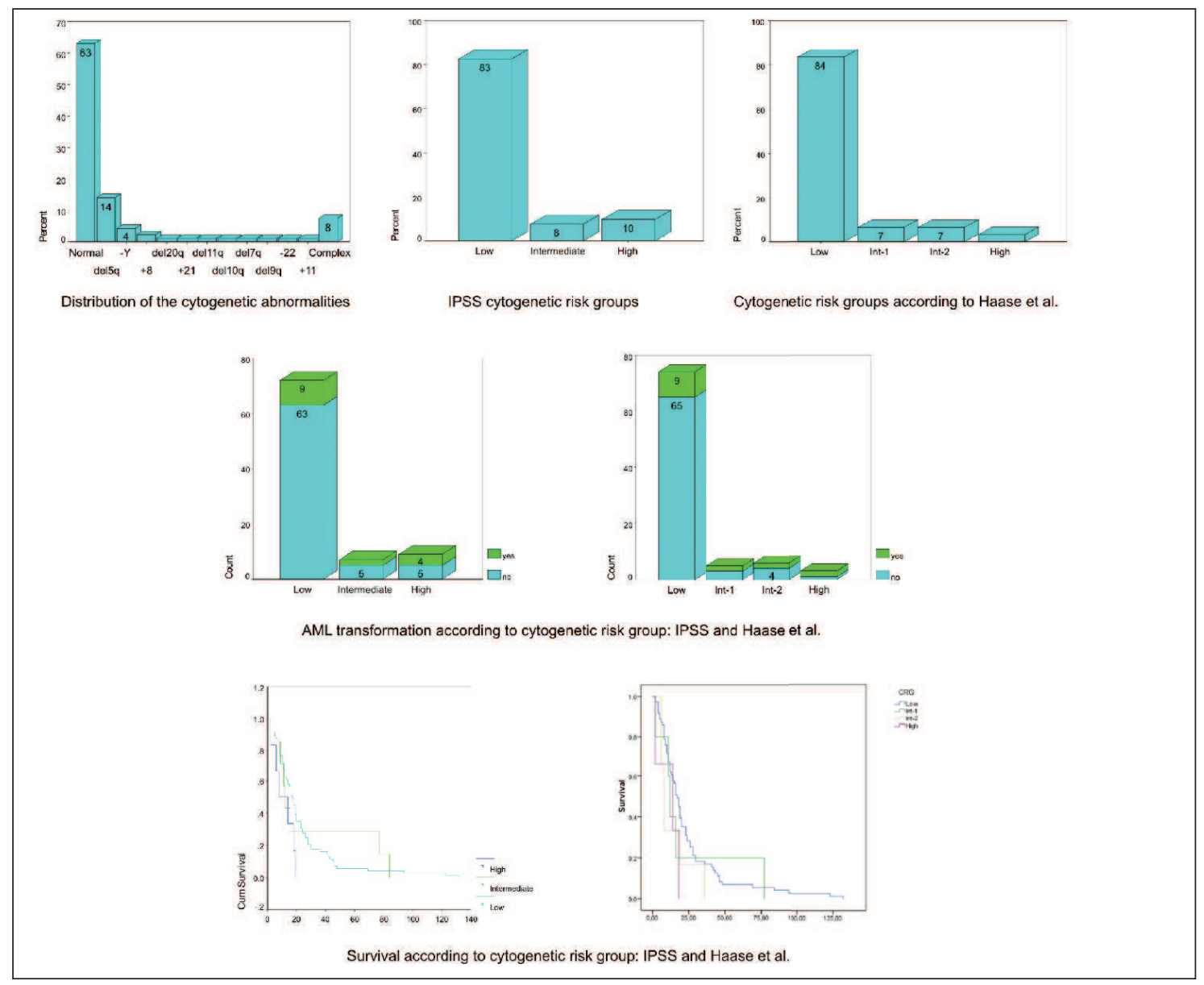

Fig. 2. Cytogenetic abnormalities and cytogenetic risk groups-correlation with survival and AML transformation

median age was 72 (20-91 years), $87 \%>60$ years of age, 52 men and 41 women. The prognostic score was determined according to IPSS, WPSS, and MDACSS. The parameters assessed were WHO type, karyotype, cytopenias, percentage of marrow blasts (morphological reassessment), age, performing status, transfusion dependence.

\section{RESULTS}

\section{Classification of MDS cases}

Patients were classified according to WHO criteria. The distribution of MDS subtypes is presented on Fig. 1: RA-29\%, RCMD-27\%, RARS5\%, RAEBI-12\%, RAEBII-11\%, 5q syndrome-11\%, MDS/MPS-2\%, MDS/fibrosis $2 \%$.

\section{Cytogenetic analysis}

Cytogenetic study was performed in $100 \%$ of patients. Abnormalities were found in $34.8 \%$, the most common-del-5q (14.6\%), -Y (4.5\%), complex karyotype (8\%), +8 (2.2\%). Cytogenetic risk groups were determined according to two different cytogenetic risk assessing scores-IPSS and Haase et al. Significant correlation was found between the cytogenetic risk groups and AML transformation and survival (Fig. 2).

\section{Risk scoring}

Tree different prognostic scoring systems were used for the assessment of the risk groups of the MDS patients. According to IPSS $41 \%$ of the patients were with low risk, $34 \%$ with Int-1, 16\%-Int-2\%, high-9\%. WPSS distributed the patients in 5 groups: 
Novel approaches in the classification and risk assessment of patients with Myelodysplastic syndromes

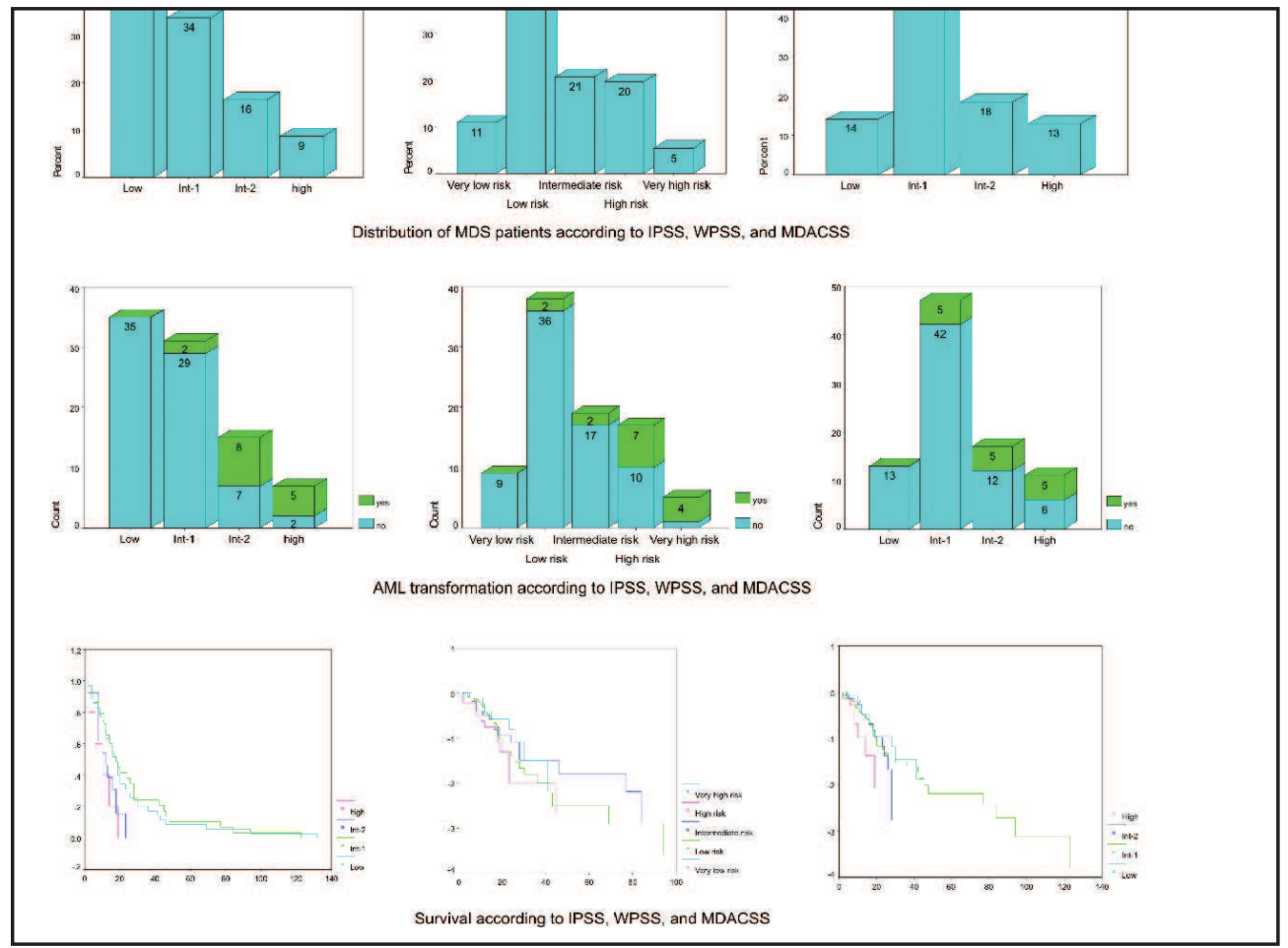

Fig. 3. MDS risk groups according to IPSS, WPSS, MDACSS-correlation with survival and AML transformation

very low-11 \%, low risk-43\%, int- $21 \%$, high-20\%, very high-5\%. According to MDACSS $14 \%$ of patients were with low, 54\%-Int-1, 18\%-Int2, 13\%-high risk. Significant correlation was found between the risk groups and AML transformation and survival in all risk stratification systems (Fig. 3)

\section{DISCUSSION}

In the present study we applied different prognostic scoring systems for risk assessment in MDS using morphological, cytogenetic, laboratory and clinical parameters. The cytogenetic risk group appeared as a very powerful prognostic factor; the risk of transformation in AML correlates significantly with the risk group. The cytogenetic prognostic system proposed by Haase et al. determines the high risk group more precisely since all patients in this group have AML progression compared to $44 \%$ in IPSS high risk group.
The three prognostic scoring systems used in this study distribute the patients in risk groups with significant difference in survival and AML transformation. However, comparison between the different scoring systems demonstrated the advantage of prognostic systems as WPSS and MDACSS, based on broader range of clinical parameters, in better distribution of intermediate risk patients and the precise determination of high and very high risk (Fig. 4).

The accurate diagnosis and risk stratification in MDS is a key to the selection of therapy, as well as to predict survival and progression in AML. Incorporation of novel prognostic markers will continue to require revision of the existing risk stratification systems. 
Ilina Micheva, Rosen Rachev, Hinco Varbanov et al.

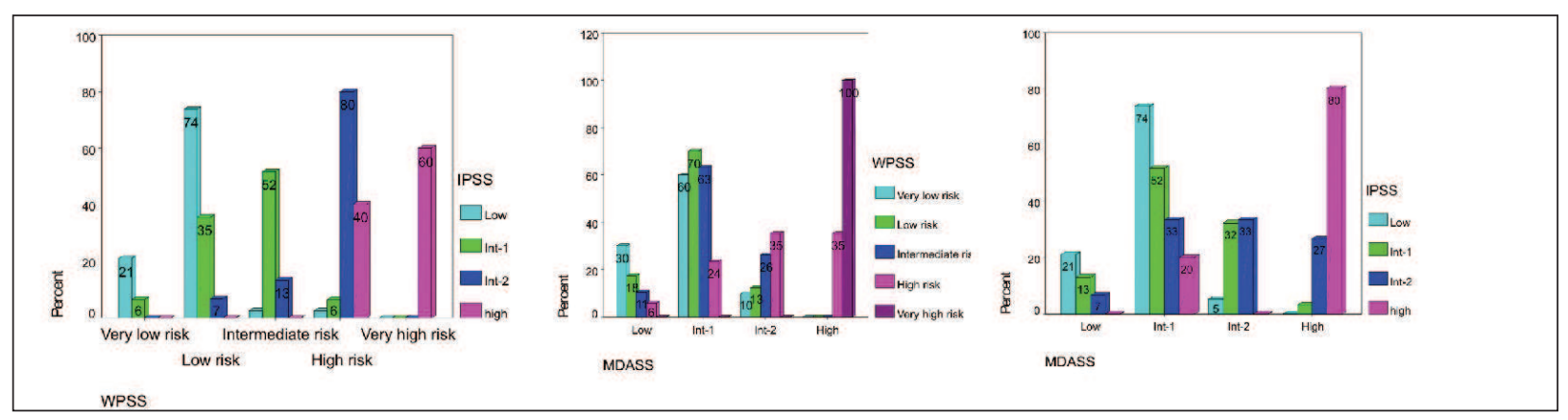

Fig. 4. Comparison between the different scoring systems-IPSS, WPSS, MDACSS

\section{REFERENCES}

1. Bejar R, Levine R, Ebert BL. Unraveling the molecular pathophysiology of myelodysplastic syndromes. J Clin Oncol. 29, 2011, 5, 504-15.

2. Bejar R, Stevenson K, Abdel-Wahab O, Galili N, Nilsson B, Garcia-Manero G, et al. Clinical effect of point mutations in myelodysplastic syndromes. N Engl J Med. 364, 2011, 26, 2496-506.

3. Bennett JM. A comparative review of classification systems in myelodysplastic syndromes (MDS). Semin Oncol. 32, 2005, 4, Suppl 5, S3-10

4. Greenberg P, Cox C, LeBeau MM, Fenaux P, Morel P, Sanz G, et al. International scoring system for evaluating prognosis in myelodysplastic syndromes. Blood. 89, 1997, 6, 2079-2088.

5. Greenberg PL, Tuechler H, Schanz J, Sanz G, Garcia-Manero G, Solé F, Bennett JM, et al. Revised international prognostic scoring system for myelodysplastic syndromes. Blood. 120, 2012,12, 2454-65.

6. Haase D, Germing U, Schanz J, Pfeilstöcker M, Nösslinger T, Hildebrandt B, et al. New insights into the prognostic impact of the karyotype in MDS and correlation with subtypes: evidence from a core dataset of 2124 patients. Blood. 110, 2007, 13, 4385-95.

7. Kantarjian H, O'Brien S, Ravandi F, Cortes J, Shan J, Bennett JM, et al. Proposal for a new risk model in myelodysplastic syndrome that accounts for events not considered in the original International Prognostic Scoring System. Cancer. 113, 2008, 6, 1351-61.

8. Malcovati L, Germing U, Kuendgen A, Della Porta MG, Pascutto C, Invernizzi R, et al. Time-dependent prognostic scoring system for predicting survival and leukemic evolution in myelodysplastic syndromes. J Clin Oncol. 25, 2007, 23, 3503-3510.

9. Schanz J, Tüchler H, Solé F, Mallo M, Luño E, Cervera J, et al. New comprehensive cytogenetic scoring system for primary myelodysplastic syndromes (MDS) and oligoblastic acute myeloid leukemia after MDS derived from an international database merge. J Clin Oncol. 30, 2012, 8, 820-829.

10. Swerdlow, S.H., Campo, E., Harris, N.L., Jaffe, E.S., Pileri, S.A., Stein, H., et al. WHO Classification of Tumours of Haematopoietic and Lymphoid Tissues, Fourth Edition EDS. WHO, 2008.

11. Walter MJ, Ding L, Shen D, Shao J, Grillot M, McLellan M, et al. Recurrent DNMT3A mutations in patients with myelodysplastic syndromes. Leukemia. 25, 2011, 7, 1153-8. 